PPPL-3243 - Preprint: March 1997, UC-427

\title{
Microinstability Properties of Negative Magnetic Shear Discharges in the Tokamak Fusion Test Reactor and DIII-D
}

\author{
G. Rewoldt \\ Plasma Physics Laboratory, Princeton University, \\ Princeton, New Jersey 08543-0451 \\ L. L. Lao \\ General Atomics \\ San Diego, California 92186-9784 \\ W. M. Tang \\ Plasma Physics Laboratory, Princeton University, \\ Princeton, New Jersey 08543-0451
}

\begin{abstract}
The microinstability properties of discharges with negative (reversed) magnetic shear in the Tokamak Fusion Test Reactor (TFTR) and DIII-D experiments with and without confinement transitions are investigated. A comprehensive kinetic linear eigenmode calculation employing the ballooning representation is employed with experimentally measured profile data, and using the corresponding numerically computed magnetohydrodynamic (MHD) equilibria. The instability considered is the toroidal drift mode (trapped-electron- $\eta_{i}$ mode). A variety of physical effects associated with differing $q$-profiles are explained. In addition, different negative magnetic shear discharges at different times in the discharge for TFTR and DIII-D are analyzed. The effects of sheared toroidal rotation, using data from direct spectroscopic
\end{abstract}




\section{DISCLAIMER}

This report was prepared as an account of work sponsored by an agency of the United States Government. Neither the United States Government nor any agency thereof, nor any of their employees, make any warranty, express or implied, or assumes any legal liability or responsibility for the accuracy, completeness, or usefulness of any information, apparatus, product, or process disclosed, or represents that its use would not infringe privately owned rights. Reference herein to any specific commercial product, process, or service by trade name, trademark, manufacturer, or otherwise does not necessarily constitute or imply its endorsement, recommendation, or favoring by the United States Government or any agency thereof. The views and opinions of authors expressed herein do not necessarily state or reflect those of the United States Government or any agency thereof. 


\section{DISCLAIMER}

Portions of this document may be illegible in electronic image products. Images are produced from the best available original document. 
measurements for carbon, are analyzed using comparisons with results from a twodimensional calculation. Comparisons are also made for nonlinear stabilization associated with shear in $E_{r} / R B_{\theta}$. The relative importance of changes in different profiles (density, temperature, $q$, rotation, etc.) on the linear growth rates is considered.

$52.55 \mathrm{Fa}, 52.35 \mathrm{Kt}, 52.35 \mathrm{Qz}$

Typeset using REVTEX 


\section{INTRODUCTION}

The properties of drift-type microinstabilities are addressed for negative (reversed) magnetic shear discharges ${ }^{1,2}$ in the Tokamak Fusion Test Reactor ${ }^{3}$ (TFTR) and DIII-D ${ }^{4}$ tokamak experiments. In particular, application of a fully kinetic linear instability calculation is made to these tokamaks. The instability studied is the electrostatic toroidal drift mode, which is destabilized by the combined effects of trapped-electron magnetic-drift precession frequency resonances and of ion temperature gradients. The theoretical tool employed here is the comprehensive kinetic toroidal microinstability code, known as the "FULL" code, described in detail in Refs. 5 and 6, which has been compared with alternative calculation methods for equivalent instability equations in Ref. 7. An approach used here is to vary the minor radius and other parameters and observe the resulting changes in the linear growth rates and real frequencies.

One question considered here is whether linear theory by itself is sufficient to account for the relatively sudden confinement transitions observed in the negative magnetic shear discharges in these two devices. This turns out not to be the case, since the linear growth rates for the instability, and the resulting quasilinear transport, are determined by the equilibrium density, temperature, $q$, and bulk plasma rotation profiles, along with the magnetohydrodynamic (MHD) equilibrium, all of which change only on the slow, transport time scale. For the cases considered, the evolution of the density and temperature profiles has more influence for the changes in the growth rate curves than the evolution of the $q$ profile. Thus, some additional effect, which allows changes on a faster time scale, has to be considered. One possibility is the nonlinear stabilization ${ }^{8,9}$ due to $E_{r} / R B_{\theta}$ shear, since $E_{r}$ can change on a faster time scale.

Changes in the q-profile associated with the introduction of negative magnetic shear affect this instability in more than one way. In addition to the direct effect of changing the magnetic shear parameter $\hat{s} \equiv(r / q) d q / d r$ on the magnetic drifts, or "bad curvature", pointed out originally by Kadomtsev and Pogutse in Ref. 10, there can be additional effects of changing the local value of $q$ itself on the mode, associated with changes in the ion Landau damping, in the Shafranov shift, and in the size of the ion sound term and therefore in the strength of the ion temperature gradient 
destabilization mechanism. The overall effect combines all of these things.

In order to roughly assess the effects of rotation on these instabilities, several different approaches are compared. An approximate toroidal rotation model derived in Refs. 11 and 12 is employed. This rotation model has associated destabilizing and stabilizing effects which are mainly due to changes in the total particle drifts resulting from the toroidal rotation and the associated centrifugal force, with the corresponding self-consistent radial electric field. In addition, stabilizing effects due to radial shearing of the eigenfunction were also observed in the two-dimensional calculation of Refs. 11 and 12, and these additional rotational effects are modeled here by means of the ballooning parameter, ${ }^{13} \theta_{0}$. Finally, the linear growth rates $\gamma$ can be compared with the nonlinear decorrelation frequency $\gamma_{E} \equiv\left|\left(R^{2} B_{\theta}^{2} / B\right) d / d \psi\left(E_{r} / R B_{\theta}\right)\right| \simeq\left|\left(R B_{\theta} / B\right) d / d r\left(E_{r} / R B_{\theta}\right)\right| \simeq$ $\left|\left(B_{\theta} / B\right) d / d r\left(E_{r} / B_{\theta}\right)\right|$ discussed in Refs. 8 and 9. It has been suggested in Ref. 8 that when $\gamma_{E} \gtrsim \gamma$, nonlinear stabilization may be sufficient to completely suppress this linearly unstable mode.

The FULL code is employed for calculating the linear eigenfrequencies and eigenfunctions of high- $n$ (toroidal mode number) toroidal instabilities, using the ballooning representation. ${ }^{13}$ These calculations focus on the lowest order in $1 / n$ ballooning representation solutions, for which the analysis is local to a single, chosen magnetic surface, though the " $1 / n$ " correction term is calculated for one case to verify that it is small. The calculation includes transit frequency resonances for untrapped particles of each species, bounce frequency and magnetic drift precession frequency resonances for trapped particles of each species, finite Larmor radius effects to all orders, and finite banana orbital dynamics. Collisions are included by means of a Krook collision operator that has been adjusted to give growth rate results close to those which would be obtained with a Lorentz operator, as described in Ref. 7. Using these eigenfrequencies and eigenfunctions, the FULL code can also calculate the corresponding quasilinear fluxes of particles and energy for each plasma species in terms of an externally-specified nonlinear saturation level. ${ }^{6}$

The calculation is interfaced with realistic, numerically calculated MHD equilibria. In general, we use finite plasma pressure/toroidal magnetic pressure $(\beta)$ numerical MHD equilibria, but employ the electrostatic version of the instability calculation for the toroidal drift branch. This 
is done because we know from prior results ${ }^{5,6}$ that the dominant finite- $\beta$ effect on the eigenfrequency comes through the MHD equilibrium, and the direct coupling effects of the perturbed electrostatic potential to the perturbed magnetic vector potential are much smaller for the present range of $\beta$ values. Also, in these calculations, the equilibrium velocity distribution function is usually taken to be a Maxwellian for all species, including the hot beam ion species and the carbon impurity species. However, a slowing-down distribution is also sometimes employed for the hot beam species. It turns out numerically to make little difference whether a Maxwellian or a slowing-down distribution is used for the beam species in these cases.

In Sec. II, the various physical effects associated with changes in the $q$-profile are explained. In Sec. III, calculation results for TFTR and DIII-D negative magnetic shear discharges are presented in the absence of plasma rotation. In Sec. IV, several different ways of including rotational effects are presented, and results are shown for several TFTR cases. Conclusions are given in Sec. V.

\section{PHYSICAL EFFECTS OF $q$-PROFILE CHANGE}

The toroidal drift mode can be destabilized either by the trapped-electron time-average toroidal magnetic precession drift resonance mechanism (collisionless trapped-electron mode mechanism) or by the ion temperature gradient (ITG or $\eta_{i}$ ) nonresonant mechanism. Accordingly, the toroidal drift mode can be stabilized completely only if both of these driving mechanisms are suppressed simultaneously. Changes in the $q$-profile, for instance from an ordinary positive magnetic shear profile (with $\hat{s}>0$ ) to a negative magnetic shear profile (with $\hat{s}<0$ ) can affect the instability in more than one way. The direct effect of changing $\hat{s}$ on the trapped-particle orbit-average magnetic drift frequency for species $j, \omega_{d j}^{(0)}$, was originally pointed out by Kadomtsev and Pogutse in Ref. 10. We denote the pitch-angle dependent part of $\omega_{d j}^{(0)}$ as $G(\kappa)$, where $\omega_{d j}^{(0)} \equiv \omega_{* j}\left(r_{n j} / R\right)\left(E / T_{j}\right) G(\kappa)$, and $\kappa$ is a pitch-angle variable that goes from zero for the most deeply-trapped particles to one at the trapped-passing boundary. $G(\kappa)$ is shown in Fig. 1 for two identical DIII-D cases, except that one case has a monotonically increasing $q$-profile $(d q / d r>0)$ and the other has negative magnetic shear $(d q / d r<0)$ at this minor radius. Positive $G(\kappa)$ corresponds to destabilizing, 


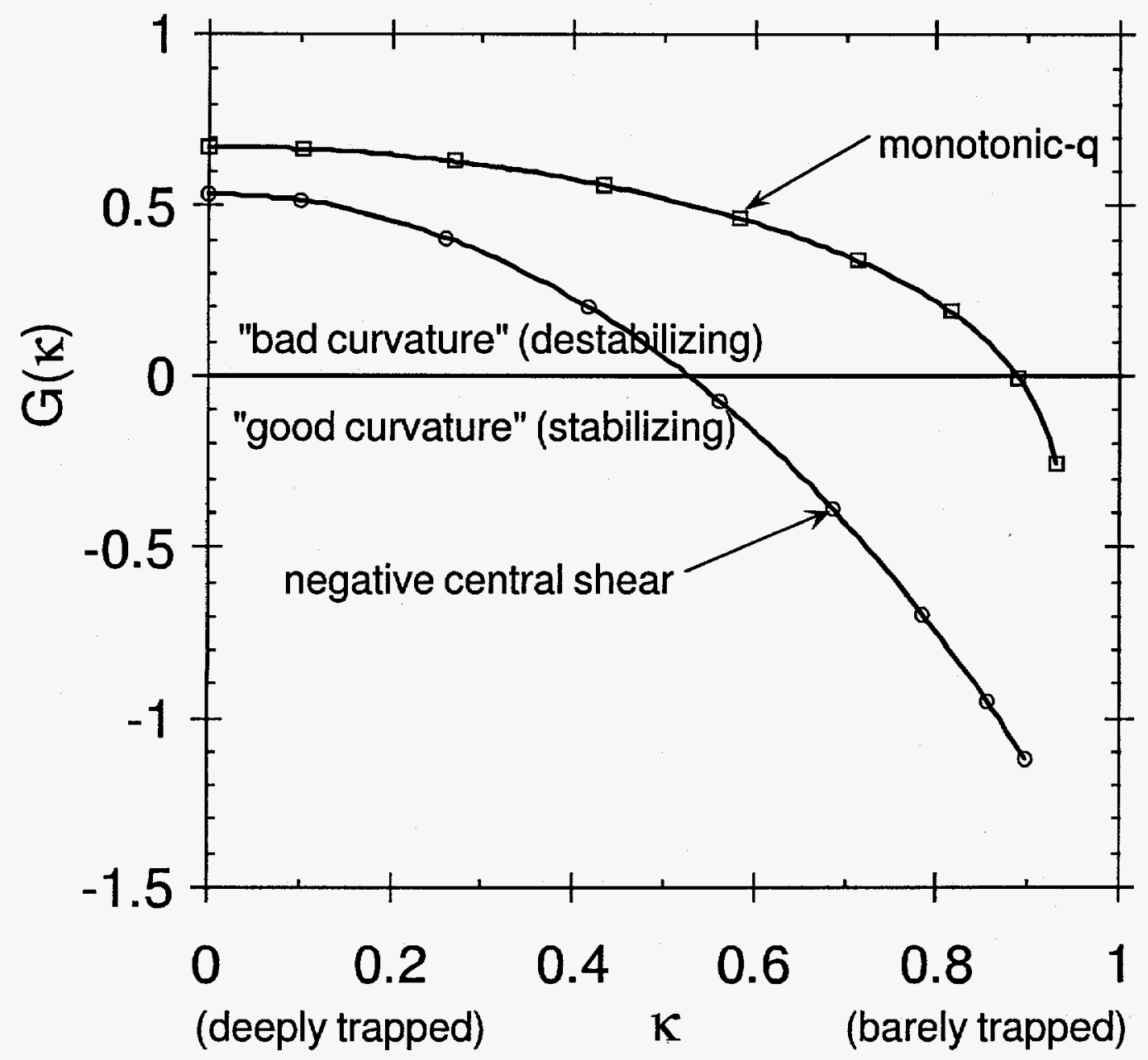

FIG. 1. Pitch-angle dependence of trapped-particle orbit-average magnetic drift frequency, for DIII-D discharge 84736 at $t=1.200 \mathrm{~s}$ and $r / a=0.300$, with numerically-calculated MHD equilibrium.

or "bad", curvature of the magnetic field lines, and negative $G(\kappa)$ corresponds to stabilizing, or "good", curvature. It can be seen in Fig. 1 that going from positive to negative $\hat{s}$ lowers the $G(\kappa)$ curve for all $\kappa$. Also, for positive $\hat{s}, G(\kappa)$ is negative only for a small fraction of barely trapped particles, while for negative $\hat{s}, G(\kappa)$ becomes negative for almost half of the trapped particles; this change for these particles is referred to as "drift reversal".

The effect of this change on the linear growth rate $\gamma$ of the toroidal drift mode is shown for a TFTR case in Fig. 2. For purposes of illustration, we temporarily employ the $\hat{s}-\alpha$ model MHD equilibrium, ${ }^{14}$ because it allows us to vary $\hat{s}$ while keeping all of the other equilibrium parameters fixed, including $q$. Here, $\gamma$ is the radially-local growth rate at lowest-order in $1 / n$ in the ballooning 


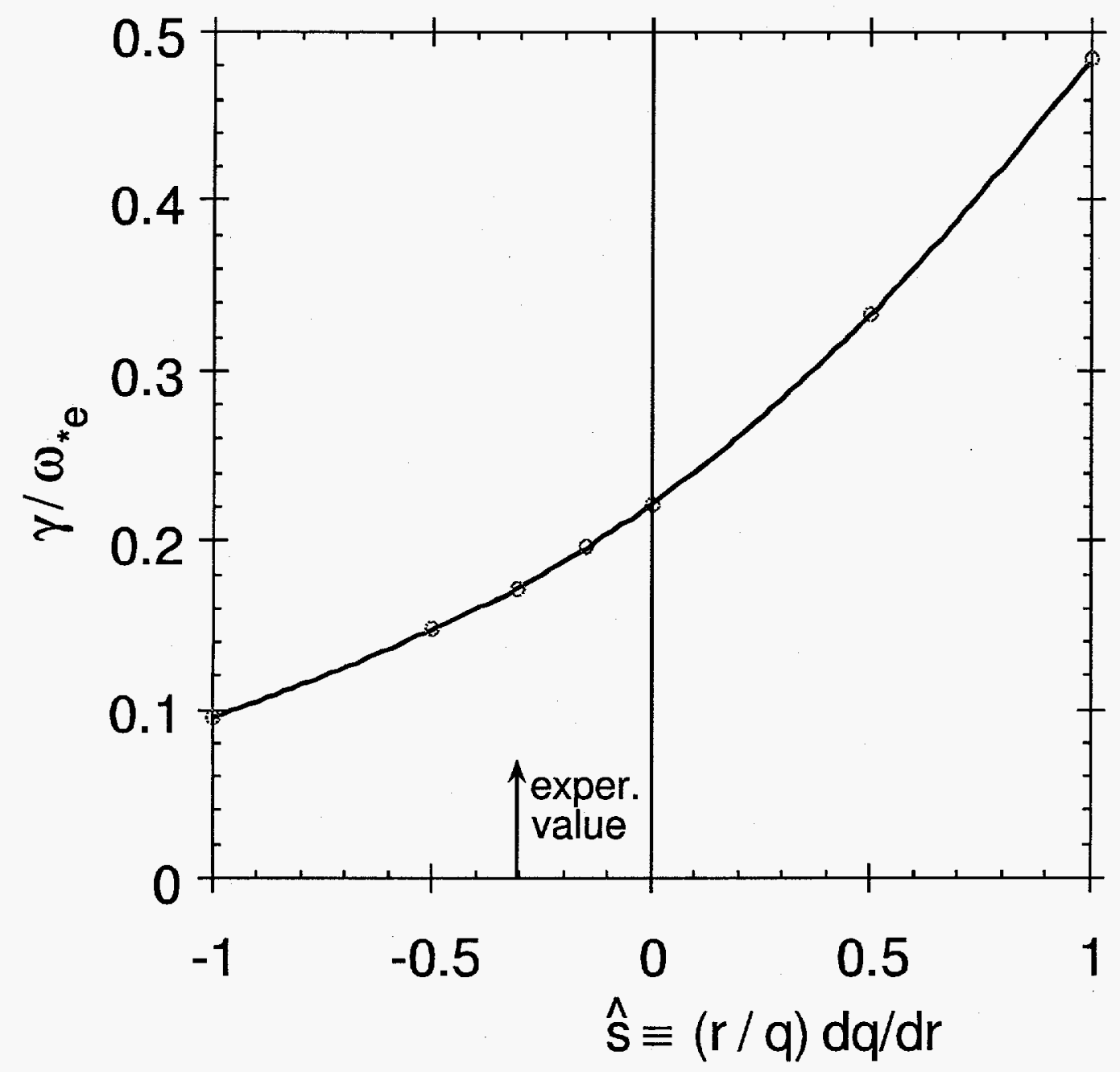

FIG. 2. Linear growth rate $\gamma$ versus magnetic shear parameter $\hat{s} \equiv(r / q) d q / d r$, for electrostatic toroidal drift mode with parameters of TFTR discharge 84011 at $t=3.00 \mathrm{~s}$ and $r / a=0.325$ with carbon and Maxwellian beam, with $k_{\theta} \rho_{i}=0.57$ and $q=2.263$, for $\hat{s}-\alpha$ model MHD equilibrium.

representation. ${ }^{13}$ It is seen that $\gamma$ decreases strongly as $\hat{s}$ decreases, due mainly to the reduction in the strength of the collisionless trapped-electron mode destabilization mechanism.

However, other physical effects on the toroidal drift mode depend on the local value of $q$, rather than on its radial derivative. First, the strength of the local ion Landau damping depends on $q$ in the following manner. When $q$ increases, $k_{\|} v_{\|}$, which can be estimated as $\omega_{t i} \equiv v_{i} / q R$ for the ions where $v_{i} \equiv\left(2 T_{i} / m_{i}\right)^{1 / 2}$, decreases. In the usual analytic limit where $\omega \gg \omega_{t i}$, this implies that the ion Landau damping will decrease, so that the linear growth rate $\gamma$ will increase. This effect is illustrated in the upper curve in Fig. 3 for the same $\hat{s}-\alpha$ model MHD equilibrium case as in 


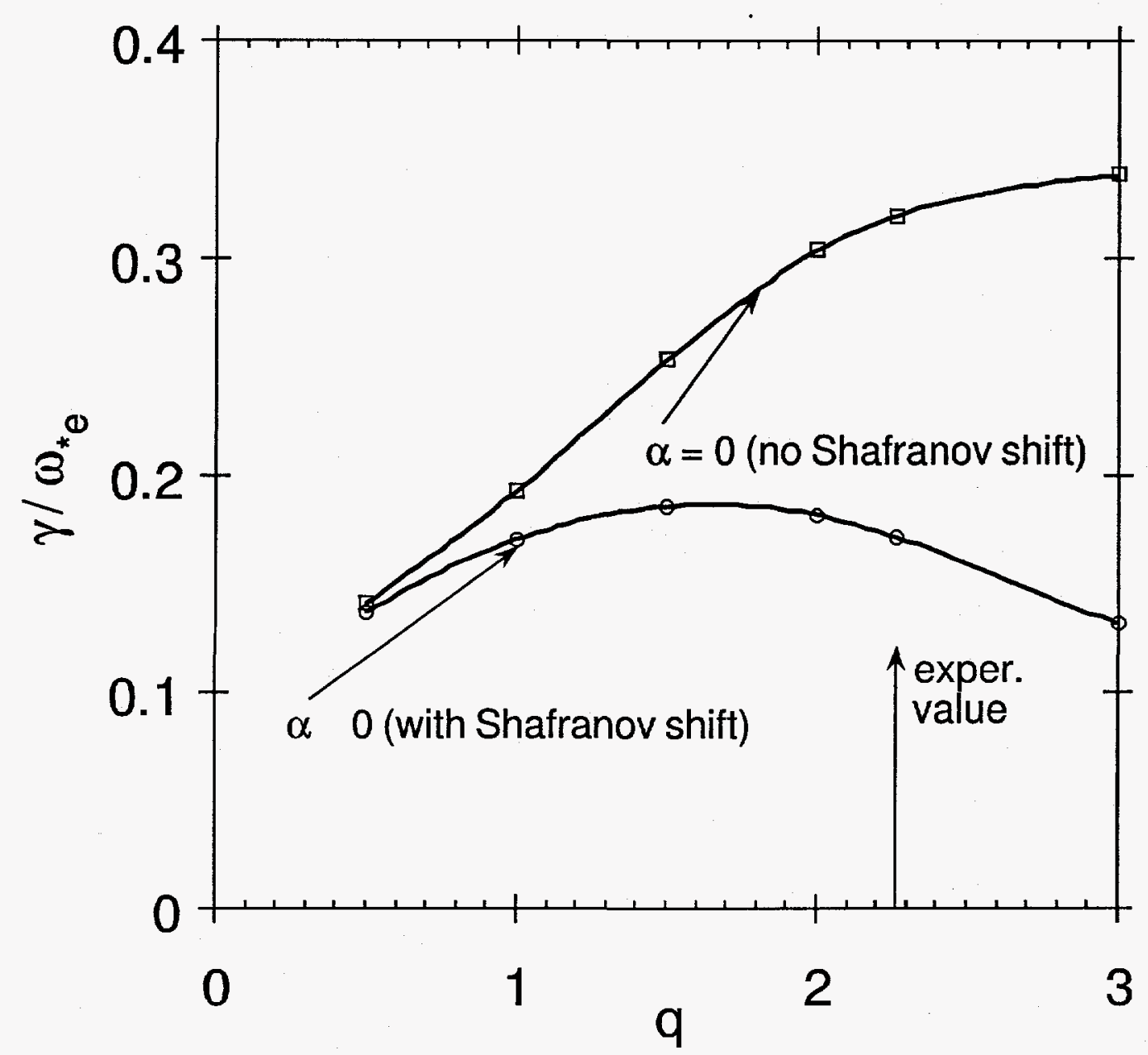

FIG. 3. $\gamma$ versus $q$, for case of Fig. 2, without $(\alpha=0)$ and with $(\alpha \neq 0)$ Shafranov shift effect, for fixed $\hat{s}=-0.3073$.

Fig. 2, but with $\alpha$ set to zero, so that the Shafranov shift effect is not included. Here, $\gamma$ is seen to increase monotonically with $q$.

Then, when nonzero values of $\alpha \equiv-q^{2} R(d \beta / d r)$ are included, increasing $q$ implies increasing values of $\alpha$, and thus of the Shafranov shift, so that the amount of "bad curvature" decreases and thus $\gamma$ decreases. This effect is illustrated in the lower curve in Fig. 3, where, above a certain value of $q$, increasing $q$ corresponds to decreasing $\gamma$.

Finally, changing $q$ can affect the strength of the ion temperature gradient destabilization mechanism directly. For increasing $q$ in the limit $\omega \gg \omega_{t i}$, this mechanism depends on the ion sound term, which is proportional to $\omega_{t i}^{2} \propto q^{-2}$, so that the term decreases, thereby decreasing the 
strength of the mechanism and decreasing the growth rate. The overall effect of changing the $q$-profile will be some combination of all of these effects.

\section{RESULTS WITHOUT ROTATION}

In both the TFTR and DIII-D experiments, confinement transitions associated with negative magnetic shear in the interior region of the plasma have been observed. These transitions take the form of relatively sudden decreases in the amount of anomalous transport in one or more of the channels (particle transport, electron energy transport, ion energy transport) to substantially below their ordinary anomalous levels. These regions can thus be referred to as "internal transport barriers". For TFTR, these are referred to as "enhanced reversed shear" (ERS) transitions, as distinguished from the "reversed shear" (RS) state with the normal anomalous transport. These ERS transitions are further classified ${ }^{15}$ for TFTR as "Type-I" transitions, where mainly the confinement of $n_{\epsilon}$ improves, and "Type- $\Pi$ " transitions, where the confinement of $T_{\epsilon}$ and $T_{i}$ improves, along with that for $n_{e}$. For DIII-D, they are referred to as "negative central shear" (NCS) transitions, ${ }^{2}$ or as the more gradual "weak or slightly negative magnetic shear" (WNS) transitions. ${ }^{16}$

An example of a TFTR Type-I ERS transition occurred in TFTR discharge 84011, over a time scale of $30 \mathrm{~ms}$ or so, from $t=2.70 \mathrm{~s}$ to $t=2.73 \mathrm{~s}$. Changes over a longer, "transport" time scale are seen after $300 \mathrm{~ms}$ at $t=3.00 \mathrm{~s}$. The corresponding experimentally-derived profiles of $T_{i}, T_{\epsilon}$, $n_{\epsilon}$, and $q$ are shown in Fig. 4 versus $r / a$. The resulting profile for $\eta_{i}^{e} \equiv\left(d \ln T_{i} / d r\right) /\left(d \ln n_{\epsilon} / d r\right)$ is also shown in Fig. 4. Results from the FULL code for the electrostatic toroidal drift mode are shown in Fig. 5. The linear growth rates $\gamma$ for this instability, maximized over $k_{\theta} \rho_{i}$ or $n$, are shown in Fig. 5(a) versus $r / a$, from a calculation including a carbon impurity species and a hot deuterium beam species with a slowing-down equilibrium distribution function. This instability normally has a stable region adjacent to the magnetic axis, but the width of this stable region can change from case to case and with time. For this TFTR discharge at $t=2.70 \mathrm{~s}$ and $t=2.73 \mathrm{~s}$, the stable region extends out to $r / a \simeq 0.125$, while for $t=3.00 \mathrm{~s}$, it has widened to $r / a \simeq 0.200$, mainly due to the decrease in $\eta_{i}^{\epsilon}$ in this region caused by the increase in $n_{e}$ and in $\left|d n_{\epsilon} / d r\right|$. 

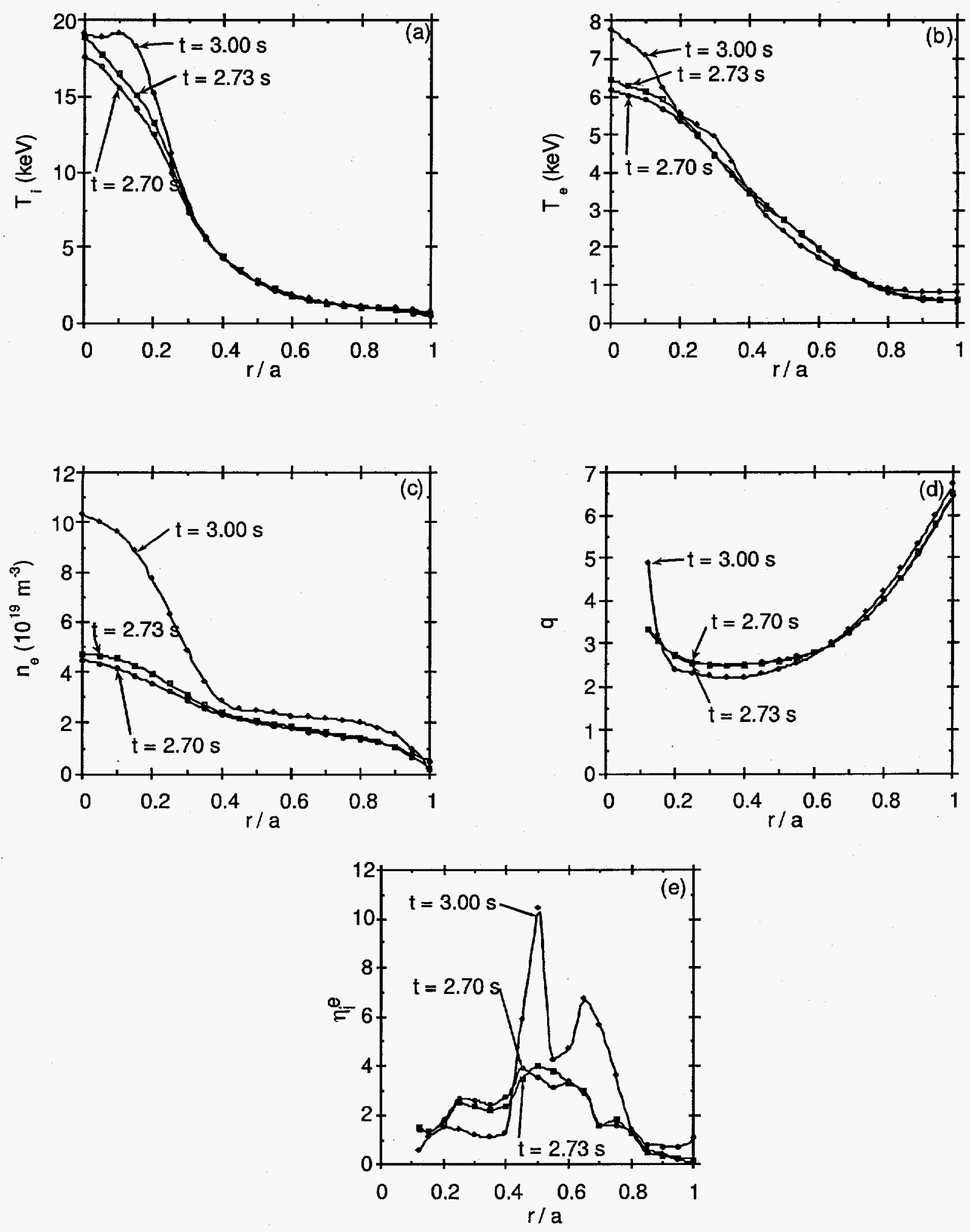

FIG. 4. Experimental profiles for TFTR Type-I ERS discharge 84011 versus $r / a$, at $t=2.70 \mathrm{~s}, 2.73 \mathrm{~s}$, and $3.00 \mathrm{~s}$, for (a) $T_{i}$, (b) $T_{\epsilon}$, (c) $n_{\epsilon}$, (d) $q$, and (e) $\eta_{i}^{\epsilon} \equiv\left(d \ln T_{i} / d r\right) /\left(d \ln n_{\epsilon} / d r\right)$. 

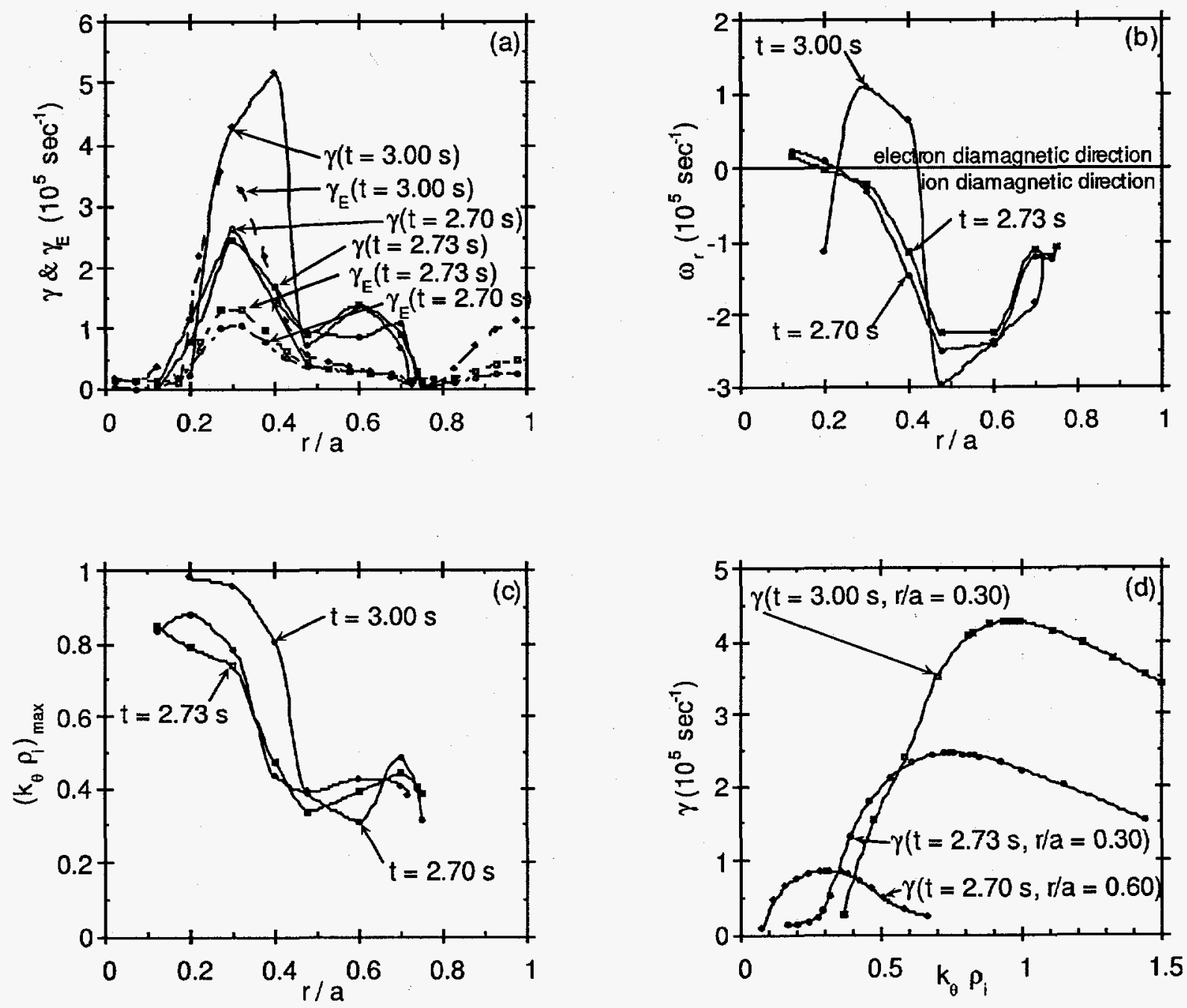

FIG. 5. Results from FULL code for electrostatic toroidal drift mode with carbon and slowing-down beam, with $\gamma$ maximized over $k_{\theta} \rho_{i}$ or $n$, for cases of Fig. 4, for (a) $\gamma$ and $\gamma_{E}=\left|\left(B_{\theta} / B\right) d / d r\left(E_{r} / B_{\theta}\right)\right|$, (b) real frequency $\omega_{r}$, and (c) $\left(k_{\theta} \rho_{i}\right)_{\max }$, versus $r / a$, and (d) $\gamma$ versus $k_{\theta} \rho_{i}$ at selected radii 
However, for $r / a>0.200, \gamma$ increases from $t=2.73 \mathrm{~s}$ to $t=3.00 \mathrm{~s}$, due to the increase of the ion temperature gradient in this region, as seen in Fig. 4(a). The corresponding curves for the nonlinear decorrelation frequency $\gamma_{E}$ at the three times are also shown in Fig. 4(a). $\gamma_{E} \gtrsim \gamma$ only out to $r / a \sim 0.15$ for $t=2.70 \mathrm{~s}$ and $t=2.73 \mathrm{~s}$, while $\gamma_{e} \gtrsim \gamma$ out to $r / a \sim 0.25$ for $t=3.00$ s. Thus, this nonlinear stabilization may cause some moderate additional widening of the stable regions.

The corresponding real frequencies $\omega_{r}$ for the instability are shown in Fig. 5(b). At $t=2.70$ $\mathrm{s}$ and $t=2.73 \mathrm{~s}$, the real frequency is mainly in the ion diamagnetic direction due to the large value of $\eta_{i}^{\epsilon}>\left(\eta_{i}^{\epsilon}\right)_{c r i t}$. However, for $r / a \lesssim 0.2, \omega_{r}$ is in the electron diamagnetic direction because $\eta_{i}^{\epsilon}<\left(\eta_{i}^{\epsilon}\right)_{\text {crit }}$ there. The toroidal drift mode can be destabilized by the ion temperature gradient mechanism only for $\eta_{i}^{e}>\left(\eta_{i}^{e}\right)_{c r i t}$, while it can be destabilized by the trapped-electron toroidal precession resonance mechanism for any value of $\eta_{i}^{\epsilon} ;\left(\eta_{i}^{\epsilon}\right)_{c r i t}$ can be roughly estimated ${ }^{17}$ as being around 1.5, though it depends on other parameters. At the later time, $t=3.00 \mathrm{~s}, \eta_{i}^{\epsilon}$ has decreased by a factor of two for $0.2<r / a<0.4$ and $\omega_{r}$ has correspondingly moved into the electron diamagnetic direction there.

The values of $k_{\theta} \rho_{i}$ that maximize $\gamma,\left(k_{\theta} \rho_{i}\right)_{\text {max }}$, are shown in Fig. 5(c). $\left(k_{\theta} \rho_{i}\right)_{\max }$ depends on many parameters, but generally it tends to increase when $\eta_{i}^{\epsilon}$ decreases, and vice-versa. Here, $\left(k_{\theta} \rho_{i}\right)_{\max }$ is of order 0.8 to 1.0 for smaller radii, where $\eta_{i}^{e}$ is smaller, and of order 0.3 to 0.4 for larger radii, where $\eta_{i}^{\epsilon}$ is larger. The dependence of $\gamma$ on $k_{\theta} \rho_{i}$ is shown in Fig. 5(d) for a selected radius at each time.

The " $1 / n$ " correction term in the ballooning representation is calculated from the formula ${ }^{13}$ $\delta \omega=\left(1 / 2 n q^{\prime}\right)\left[\left(\partial^{2} \omega / \partial r^{2}\right)\left(\partial^{2} \omega / \partial \theta_{0}^{2}\right)\right]^{1 / 2}$, where $\omega\left(r, \theta_{0}\right)$ is the lowest order mode frequency. Here, the derivatives are evaluated at $r=r_{0}$ and ballooning parameter $\theta_{0}=\theta_{0}^{0}$, the values which maximize $\gamma$. For $n=41$ or $k_{\theta} \rho_{i}=0.67, r_{0} / a=0.325$ and $\theta_{0}^{0}=0$, as expected for an up-down symmetric equilibrium. At this radius, $\hat{s} \simeq-0.036$. Evaluating the derivatives numerically gives $\delta \omega=(-0.140-0.093 i) \times 10^{5} \mathrm{~s}^{-1}$, compared to $\omega\left(r_{0}, \theta_{0}^{0}\right)=(+0.608+2.951 i) \times 10^{5} \mathrm{~s}^{-1}$, so that $|\delta \omega| /|\omega|=0.056$. Thus, omitting the " $1 / \mathrm{n}$ " correction term even in this case where $\hat{s}$ is small still gives an acceptably small error, and it will be omitted in the other cases in this work as well. 

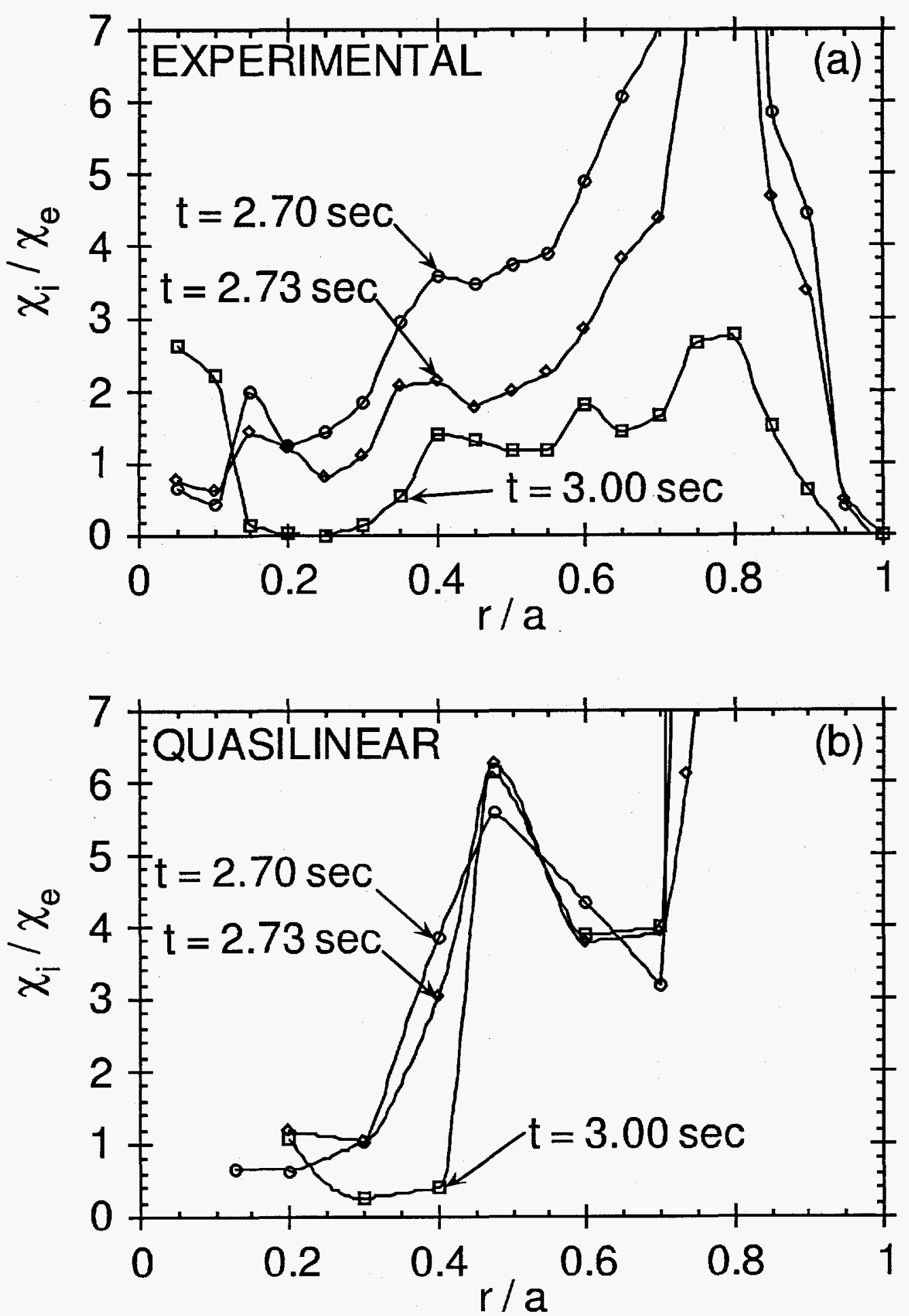

FIG. 6. (a) Experimental and (b) quasilinear ratios $\chi_{i} / \chi_{e}$ versus $r / a$ for cases of Figs. 4 and 5. 
Using these results for the eigenfrequencies and the associated eigenfunctions, the FULL code calculates the particle flux $\Gamma_{j}$ and the total energy flux $Q_{j}$ for each species $j$ for this value of $k_{\theta} \rho_{i}$ or $n$. From these fluxes we construct the thermal diffusivities $\chi_{\epsilon} \equiv-\left[Q_{e}-(3 / 2) \Gamma_{\epsilon}\right] /\left[n_{\epsilon} d T_{e} / d r\right]$ and $\chi_{i} \equiv-\sum_{j}\left[Q_{j}-(3 / 2) \Gamma_{j}\right] /\left[\sum_{j} n_{j} d T_{j} / d r\right]$, where the summation is over all thermal ion species, which are the background deuterium ions and the carbon impurity ions for the present case. These definitions are chosen to match those used in the "TRANSP" experimental data analysis code. ${ }^{18}$ While the quasilinear $\chi_{e}$ and $\chi_{i}$ individually depend on the nonlinear saturation level, the ratio $\chi_{i} / \chi_{e}$ is independent of the saturation level, and can be compared to the corresponding experimental ratio computed from the TRANSP code results. The experimental ratios are shown in Fig. 6(a) and the quasilinear ratios are shown in Fig. 6(b) for the three times. Concentrating on the radial region $0.2<r / a<0.4$, the experimental ratio has a noticeable decrease from $t=2.70 \mathrm{~s}$ to $t=2.73 \mathrm{~s}$, and then drop to a level consistent with zero at $t=3.00 \mathrm{~s}$. The quasilinear ratio, however, can only respond substantially on a transport time scale because it is determined by the profile input data, which changes on that time scale. Thus, the quasilinear ratio is generally the same in this radial region for $t=2.70 \mathrm{~s}$ and $t=2.73 \mathrm{~s}$, but then decreases to a value that is small but nonzero by $t=3.00 \mathrm{~s}$. This is mainly due to the decrease in $\eta_{i}^{e}$ in this region, from being larger than $\left(\eta_{i}^{e}\right)_{c r i t}$ for $t=2.70 \mathrm{~s}$ and $t=2.73 \mathrm{~s}$ to being slightly less than $\left(\eta_{i}^{e}\right)_{c r i t}$ at $t=3.00$ s. It is known from previous results ${ }^{6}$ that $\chi_{i}$ is normally the dominant transport coefficient for $\eta_{i}^{e}>\left(\eta_{i}^{e}\right)_{c r i t}$, while $\chi_{e}$ is normally the dominant transport coefficient for $\eta_{i}^{e}<\left(\eta_{i}^{e}\right)_{c r i t}$. Thus, the change in the quasilinear ratio for the present case is in the expected direction. Also, the electronion energy exchange term implemented in the TRANSP code is approximate, so the experimental ratio is correspondingly uncertain. Over the transport time scale, the quasilinear ratio reproduces most of the change seen in the experimental ratio in this radial region where the internal transport barrier has formed. However, the quasilinear ratio change can not account properly for the experimental change on the faster $30 \mathrm{~ms}$ time scale.

A useful comparison can be made of "matched" TFTR discharges which start out being almost identical, but one discharge (88299) exhibits a Type-I ERS confinement transition, while the other (88298) does not. These two discharges are compared from an experimental point of view in 

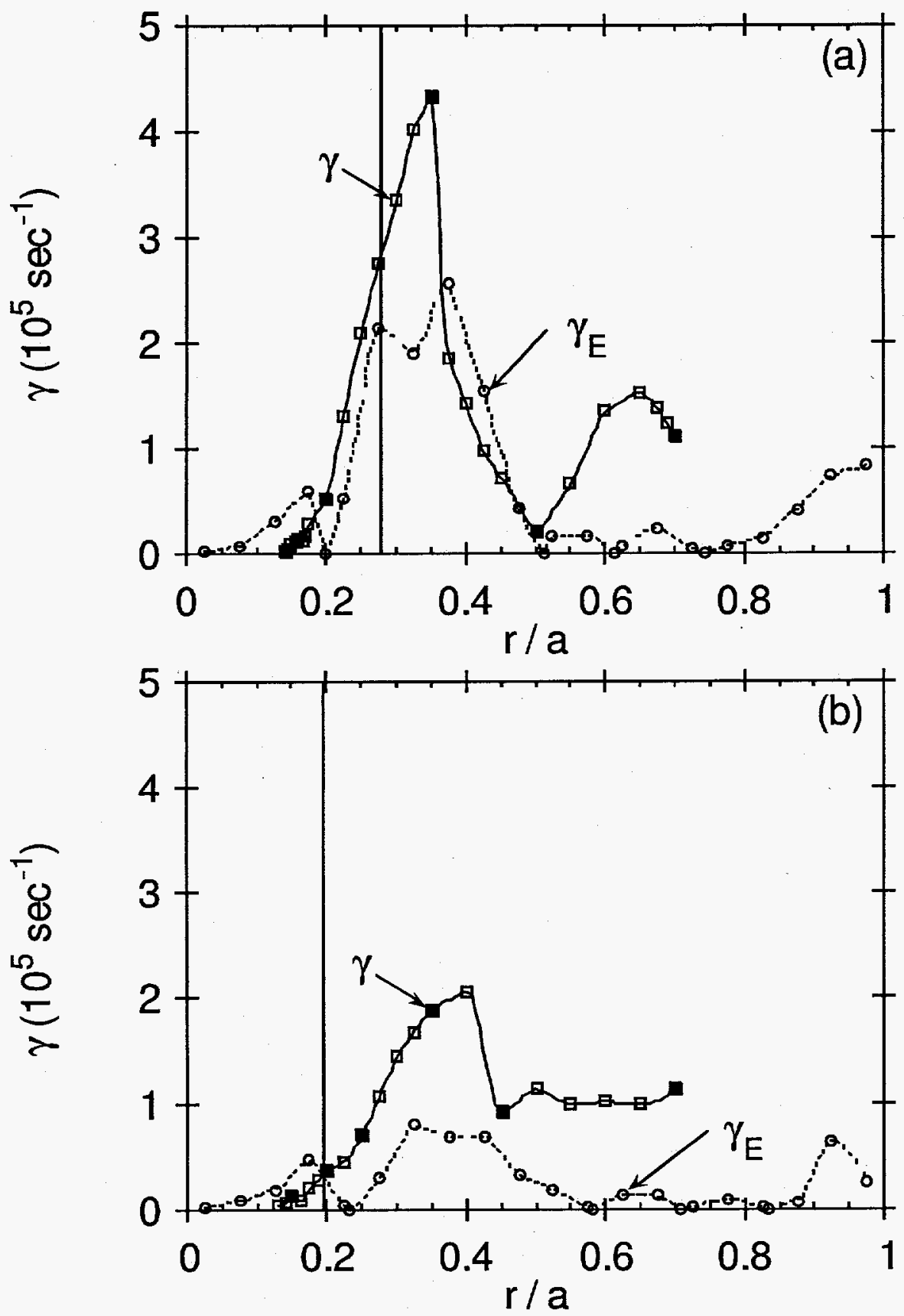

FIG. 7. Results for $\gamma$ and $\gamma_{E}$ versus $r / a$ for TFTR (a) Type-I ERS discharge 88299 and (b) non-ERS discharge 88298 , at $t=2.75 \mathrm{~s}$, with $\gamma$ for electrostatic toroidal drift mode with carbon and Maxwellian beam, (maximized over $k_{\theta} \rho_{i}$ at solid points). 
Ref. 19, including results of fluctuation measurements in the two discharges, showing a very low level of fluctuations over a larger radial distance from the magnetic axis in the ERS discharge than in the non-ERS discharge. Results have been obtained from the corresponding FULL code calculations for the electrostatic toroidal drift mode, including a carbon impurity species and a hot deuterium beam species with a Maxwellian distribution function, with $\gamma$ maximized over $k_{\theta} \rho_{i}$ or $n$. For $t=2.75 s$ (just after the ERS transition time in the ERS discharge), $\gamma$ is shown in Fig. 7(a) for the discharge that did have the ERS transition, and in Fig. 7(b) for the discharge that did not have the transition. $\gamma$ is actually larger in the ERS shot, but $\gamma_{E}$ is also larger, and $\gamma_{E}$ tracks along with $\gamma$ out to $r / a \sim 0.27$, while for the non-ERS discharge, $\gamma_{E} \gtrsim \gamma$ only out to $r / a \sim 0.19$, and further out $\gamma_{E}$ becomes much smaller than $\gamma$. This presumably wider stabilized region for the ERS discharge correlates with the wider experimentally-observed region of low fluctuation level.

Another type of ERS confinement transition which takes place in TFTR is the Type-II transition, where not just $n_{\epsilon}$, but also $T_{\epsilon}$ and $T_{i}$, start increasing strongly near the plasma center at or after the ERS transition time, as seen in TFTR discharge 91222. Results were obtained from the FULL code for the electrostatic toroidal drift mode including a carbon impurity species and a Maxwellian hot beam species for $t=3.20 \mathrm{~s}$, the ERS transition time. Results for $\gamma, \omega_{r}$, and $\gamma_{E}$, for $\gamma$ maximized over $k_{\theta} \rho_{i}$, are shown in Fig. 8(a) and for $\eta_{i}^{e}$ and $\left(k_{\theta} \rho_{i}\right)_{\max }$ in Fig. 8(b). These results show a strongly unstable region for $0.1<r / a<0.7$, whose width is not appreciably affected by $\gamma_{E}$. Thus, for this Type-II transition, the combination of $\gamma$ and $\gamma_{E}$ does not appear to be sufficient to account for this transition. This suggests that the mechanism for TFTR Type-II transitions may be different in some qualitative sense from that for Type-I transitions.

Confinement transitions of a somewhat different type are seen in DIII-D. After the NCS transition for DIII-D discharge 84736, the confinement of $T_{i}$ has a large increase, along with some change in the shape of the $T_{i}$ profile, while $T_{\varepsilon}$ and $n_{e}$ only have much smaller increases. In particular, before the transition at $t=1.0 \mathrm{~s}, T_{i}$ decreases strongly with radius from the magnetic axis, while after the transition at $t=1.2 \mathrm{~s}, T_{i}$ has a flat region next to the magnetic axis and decreases strongly further out. Results from the FULL code for the electrostatic toroidal drift mode with a carbon impurity species and a Maxwellian hot beam species are shown in Fig. 9(a) for $k_{\theta} \rho_{i} \simeq 0.5$, 

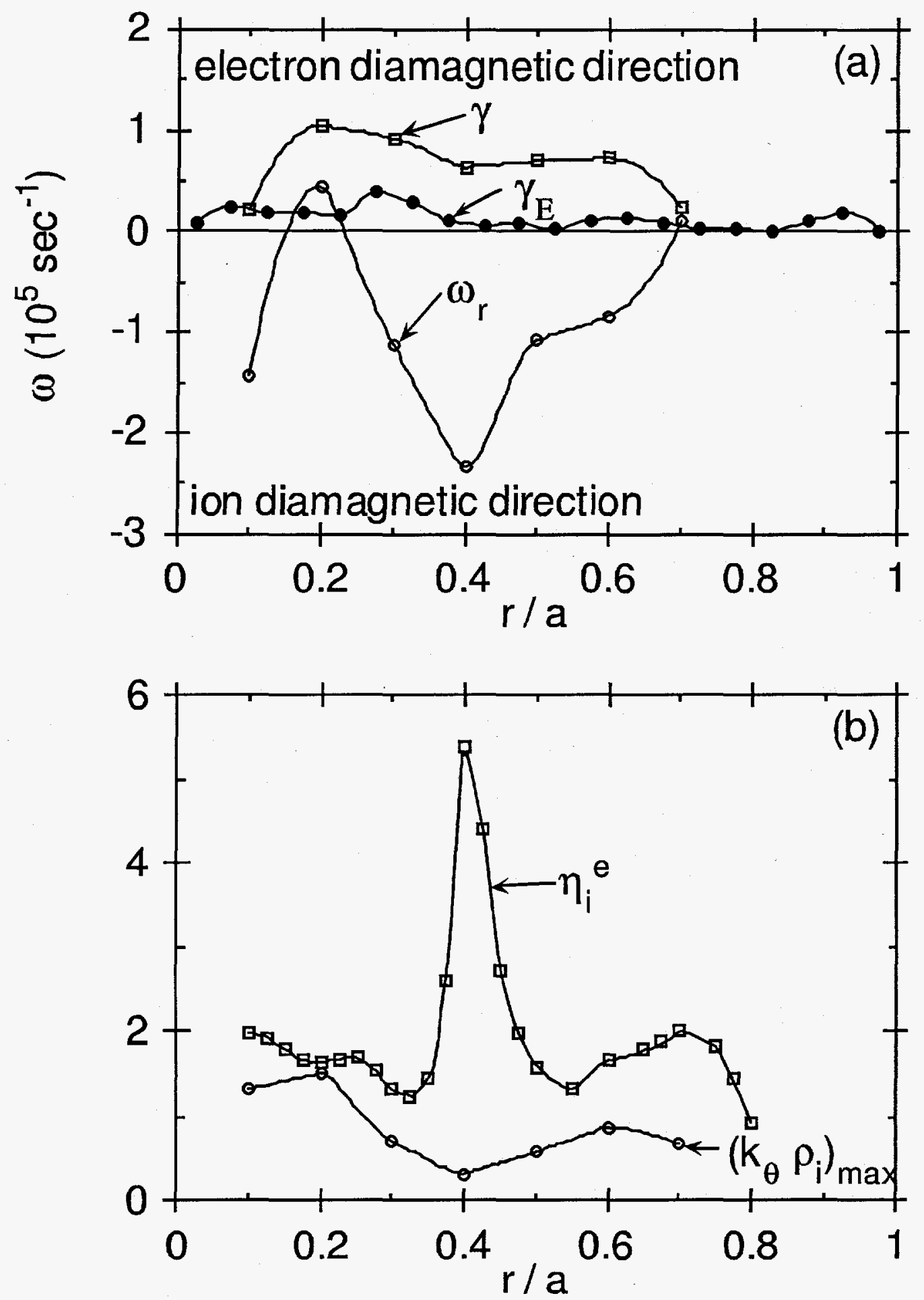

FIG. 8. (a) Results for $\gamma$ and $\gamma_{E}$ versus $r / a$ for TFTR Type-II ERS discharge 91222 and (b) corresponding values of $\left(k_{\theta} \rho_{i}\right)_{\max }$ and $\eta_{i}^{\varepsilon}$, at $t=3.20 \mathrm{~s}$, with $\gamma$ for electrostatic toroidal drift mode with carbon and Maxwellian beam, with $\gamma$ maximized over $k_{\theta} \rho_{i}$. 

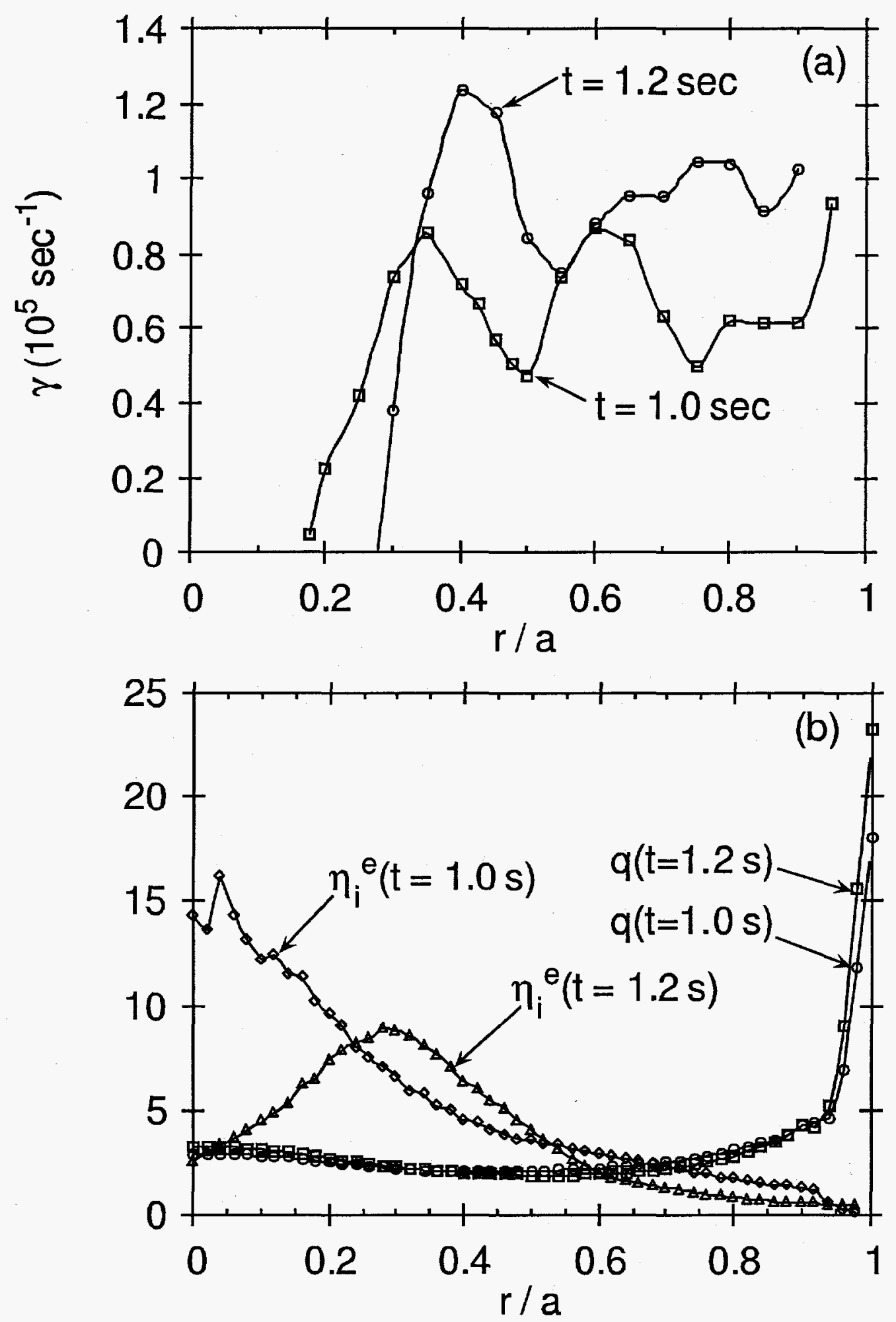

.FIG. 9. (a) Results for $\gamma$ versus $r / a$ for DIII-D NCS discharge 84736 and (b) corresponding values of $q$ and $\eta_{i}^{e}$, at $t=1.0 \mathrm{~s}$ and $1.2 \mathrm{~s}$, with $\gamma$ for electrostatic toroidal drift mode with carbon and Maxwellian beam, with $k_{\theta} \rho_{i}=0.47$. 

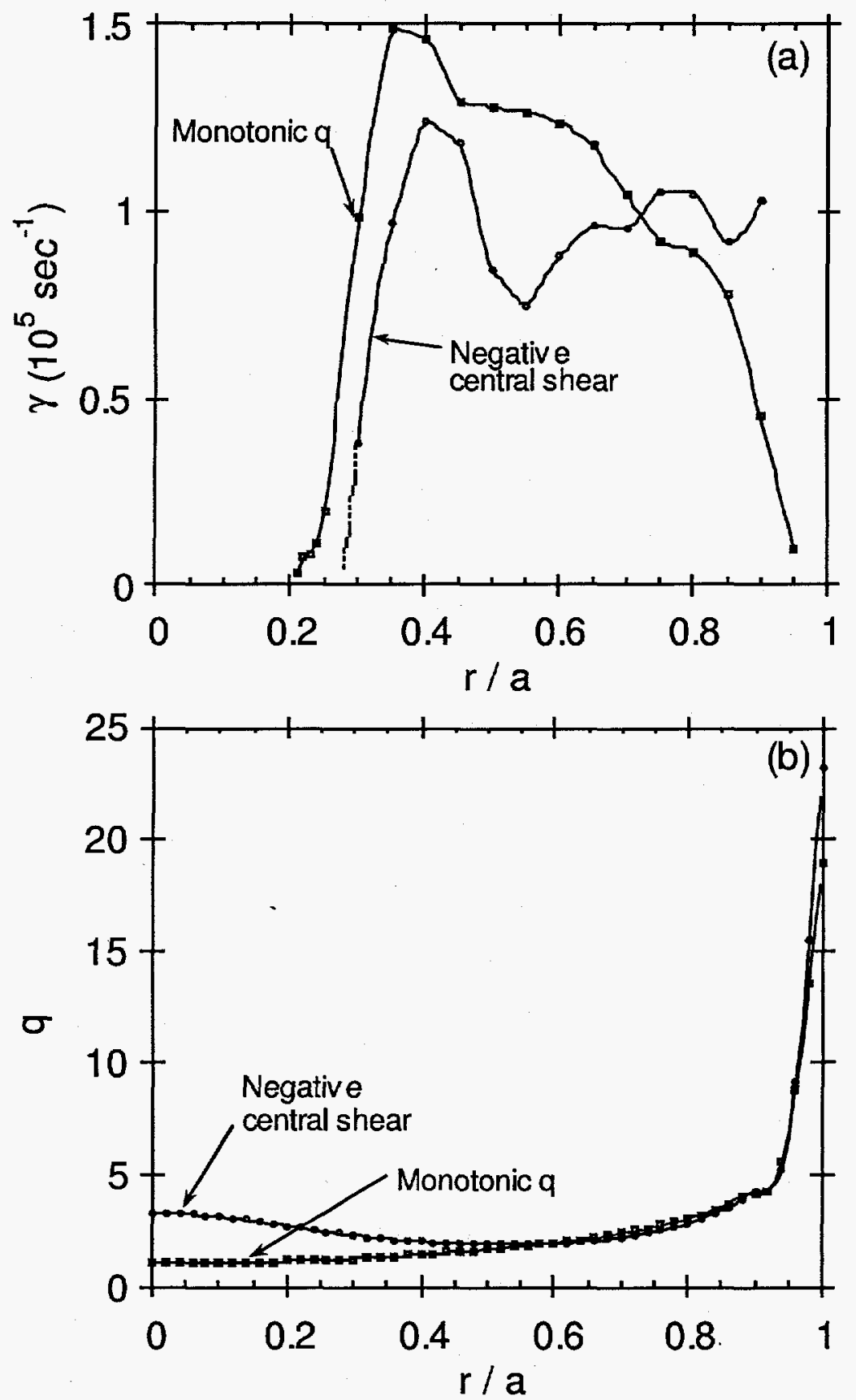

FIG. 10. (a) Results for $\gamma$ versus $r / a$ for case of Fig. 9 at $t=1.2 \mathrm{~s}$, and for same case but with artificial monotonic $q$ profile. (b) corresponding $q$ profiles. 
the value which maximizes $\gamma$ for $r / a=0.50$. Fig. 9(b) shows the corresponding profiles of $\eta_{i}^{e}$ and $q$. Due to the change in the $T_{i}$ profile, the maximum in $\eta_{i}^{e}$ moves outward, and the value of $\eta_{i}^{\epsilon}$ decreases for $r / a<0.2$. The linear growth rate $\gamma$ decreases for $r / a<0.3$, mainly due to the changes in the density and temperature profiles. The $q$ profile changes very little over this 200 ms interval, and thus contributes very little to the change in the growth rate curve. However, the moderately negative magnetic shear for $r / a<0.4$ is somewhat stabilizing, as can be seen from Fig. 10, where results for an artificial case for $t=1.2 \mathrm{~s}$ with a monotonically increasing $q$-profile are compared with results for the actual negative central shear profile. The stable region adjacent to the magnetic axis is slightly wider for the negative central shear case. This DIII-D discharge is discussed in an experimental context in Ref. 16. As shown in Fig. 5 of Ref. 16, additional width of the stabilized region, due to the increase in $\gamma_{E}$ with time, is needed to explain the increase in width of the region in which the experimental total thermal conductivity $\chi$ is reduced.

Another type of discharge obtained in DIII-D is the WNS discharge, which is also discussed from an experimental point of view in Ref. 16. For WNS discharge 84713 at $t=2.08 \mathrm{~s}$, results have been obtained from the FULL code for the electrostatic toroidal drift mode with a carbon impurity species and a Maxwellian hot beam species for $k_{\theta} \rho_{i}=0.49$, the value which maximizes $\gamma$ at $r / a=0.70$. The $\gamma, \omega_{r}$, and $\gamma_{E}$ profiles are shown in Fig. 11(a) and the corresponding $n_{\epsilon}$ and $q$ profiles in Fig. 11(b). The H-mode-like density profile is almost flat for $r / a<0.8$; in this region $\eta_{i}^{\epsilon}$ is extremely large and $\omega_{r}$ is correspondingly in the ion diamagnetic direction. In the edge region, $r / a>0.8$, the density gradient becomes large, $\eta_{i}^{e}$ is small, and the real frequency is correspondingly in the electron diamagnetic direction. The central stable region for this case extends out from the magnetic axis to $r / a \simeq 0.5$. Note that $\gamma_{E}>\gamma$ for all radii, suggesting overall stabilization of this instability. This is consistent with the experimental finding (cf. Fig. 11 of Ref. 16) that $\chi_{i}$ is of the order of the standard neoclassical $\chi_{n e o}$ throughout the plasma interior. 

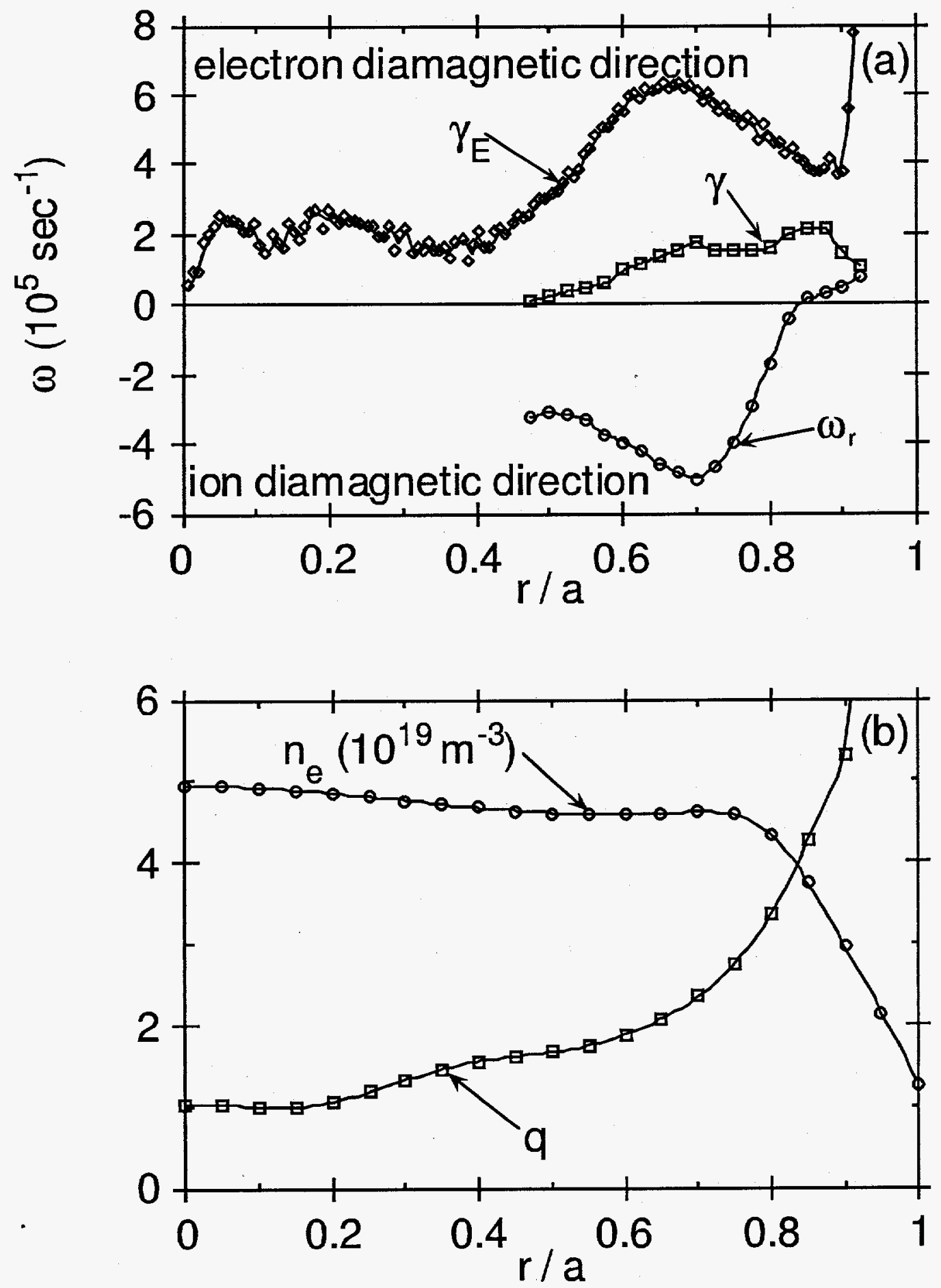

FIG. 11. Results for (a) $\gamma, \omega_{r}$, and $\gamma_{E}$ and (b) $q$ and $n_{\epsilon}$, versus $r / a$ for DIII-D WNS discharge 84713 at $t=2.08 \mathrm{~s}$, for electrostatic toroidal drift mode with carbon and Maxwellian beam, for $k_{\theta} \rho_{i}=0.49$. 


\section{ROTATIONAL EFFECTS}

The effects of sheared toroidal plasma rotation are estimated here by means of an approximate toroidal rotation model derived in Refs. 11 and 12. In this model, the toroidal rotation causes different centrifugal forces for the ions and electrons, which are balanced by an electric field. The $E \times B$ drift associated with this electric field gives additional terms which add on to the usual magnetic curvature and gradient drift frequency terms, which are both linear and quadratic in the toroidal rotation frequency, and which can be either stabilizing or destabilizing. The additional drift terms include one proportional to the shear in the toroidal rotation frequency. Also, in the resonant numerator, along with the density gradient and temperature gradient terms, an additional (Kelvin-Helmholtz) term proportional to the toroidal rotation frequency shear appears.

This rotation model makes a number of significant approximations. First, in the usual radial force balance expression, $E_{r}=V_{\phi} B_{\theta}-V_{\theta} B_{\phi}+\left(1 / n_{i} e_{i}\right) d p_{i} / d r$, this rotation model only accounts for the first, toroidal rotation, term and effectively omits the poloidal rotation term (which is experimentally observed to be small in the plasma interior) and the ion pressure gradient term. Second, the rotation model assumes the same toroidal rotation frequency for all species. Third, the rotation model assumes a model toroidal geometry with circular, concentric, large-aspect-ratio magnetic surfaces, whereas all of the rest of the instability calculation allows general, numerically-calculated magnetic surfaces of arbitrary cross-sectional shape and aspect ratio. These limitations are not expected to change the overall rotational effect in a qualitative sense. The numerically largest effect of the toroidal rotation is a Doppler shift of the real frequency of the mode. It should be emphasized that this model is for the linear effects of sheared toroidal rotation of the bulk plasma, and is distinct from the effects of the nonlinear decorrelation frequency $\gamma_{E}=\left|\left(R B_{\theta} / B\right) d / d r\left(E_{r} / R B_{\theta}\right)\right|$ discussed in Refs. 8 and 9. Also, it has been pointed out previously ${ }^{20}$ that large plasma toroidal rotation velocities invalidate the usual one-dimensional ballooning representation; however, for sufficiently small toroidal rotation velocities, the ballooning representation is still usable, according to Ref. 21.

This toroidal rotation model was first applied in the two-dimensional, low- $n$ calculation de- 
scribed in Refs. 11 and 12. Strong stabilization of electrostatic toroidal drift modes (trapped-ion modes) was seen for moderate values of the Mach number on the magnetic axis, $M_{0} \equiv V_{t o r}(r=$ $0) / v_{i}(r=0)$, where $V_{\text {tor }}$ is the local toroidal rotation velocity. For instance, Fig. 8 of Ref. 12 shows a factor of two reduction in the linear growth of the $n=4$ trapped-ion mode for normal TFTR L-mode parameters for $M_{0} \simeq \pm 0.1$. Associated with this value of the rotation is a strong "shearing" of the eigenfunction, as shown in Fig. 10 of Ref. 12. With increasing $M_{0}$, the individual eddies of the eigenfunction around the magnetic field minimum at $\theta=0$ become increasingly twisted and radially narrowed, corresponding to an increase in the local effective $k_{r}$. On the other hand, for positive (negative) $M_{0}$, the local eddies at some negative (positive) values of $\theta$ are not twisted or radially narrowed. Thus, the poloidal angle $\theta$ corresponding to the minimum effective value of $k_{r}$ in the two-dimensional eigenfunction shifts to increasingly negative (positive) values with increasing positive (negative) $M_{0}$. This effect of the radial shearing of the eigenfunction is in addition to the direct effect on the growth rate of the additional particle drifts.

This approximate toroidal rotation model has been implemented in the ballooningrepresentation FULL code in two parts. First, the additional particle drifts and the other direct effects of toroidal rotation, as described in Refs. 11 and 12, have been added. Several DIII-D monotonic- $q$ cases have been treated with this form of the rotation model in Ref. 22. Second, the effect of the radial shearing of the eigenfunction has been modeled by means of the parameter of the ballooning representation ${ }^{13}$ called the "ballooning parameter", $\theta_{0}$. In the absence of rotation, $\theta_{0}$ is set to the value which maximizes the growth rate, which for an up-down symmetric equilibrium is normally $\theta_{0}=0$. However, with toroidal rotation, $\theta_{0}$ can be used instead to model the change seen in the two-dimensional results in the effective value of $k_{r}$ with changing toroidal rotation, since, in the ballooning representation, $k_{r} \propto\left(\theta-\theta_{0}\right)$. Thus, at $\theta=0, k_{r}$ is proportional to $\theta_{0}$. In particular, we can make $\theta_{0}$ an explicit function of the local Mach number $M \equiv V_{t o r} / v_{i}$ by choosing an appropriate functional form and adjusting the constants to give a reasonably good match to the two-dimensional growth rate results.

This has been done for the $n=4$ trapped-ion mode case with TFTR L-mode parameters described in Refs. 11 and 12, resulting in the functional form $\theta_{0}=\theta_{0}(M) \equiv-6.83 M /\left[M^{2}+\right.$ 

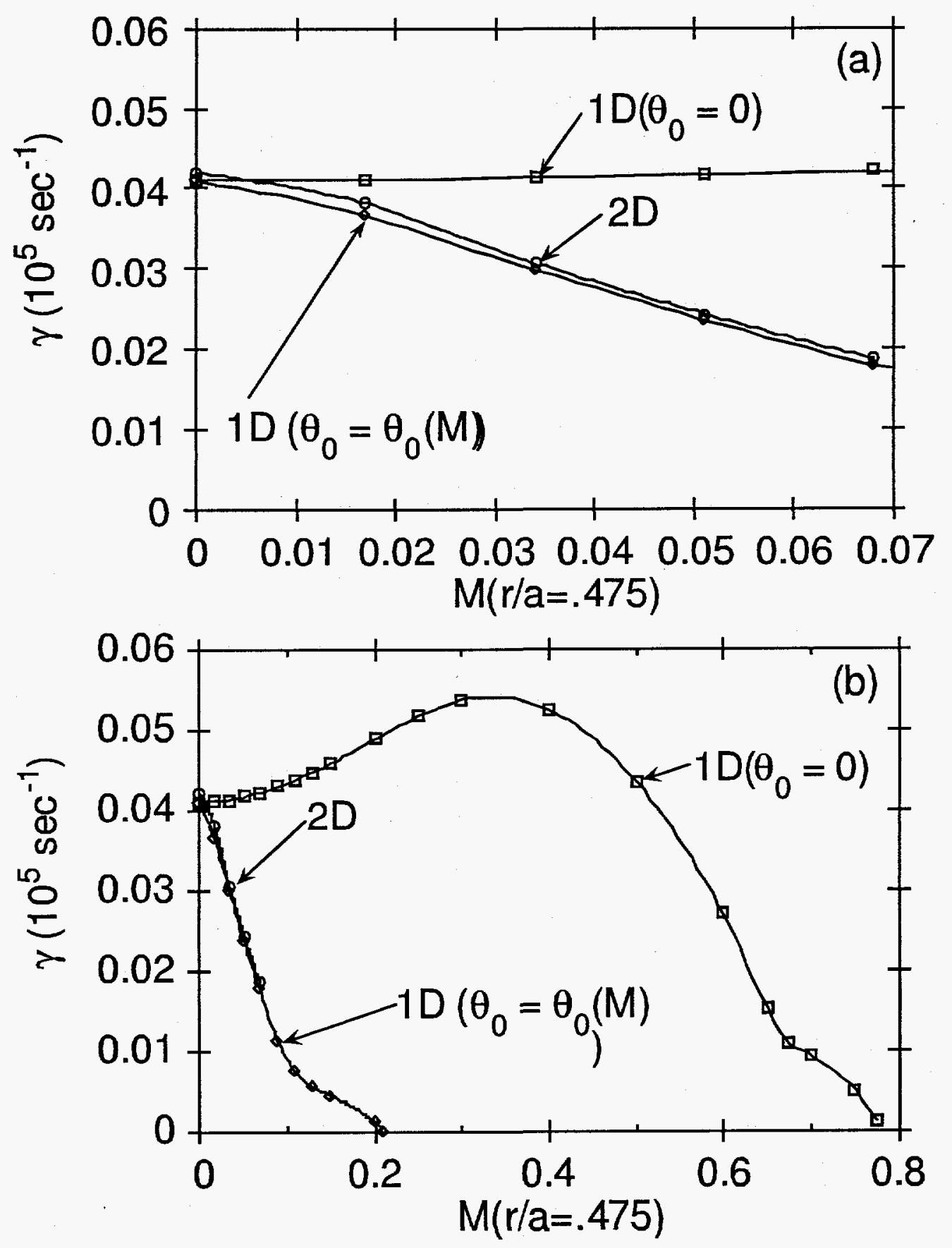

FIG. 12. Linear growth rates $\gamma$ versus local Mach number $M \equiv V_{\text {tor }} / v_{i}$ at $r / a=0.475$ for $n=4$ electrostatic trapped-ion mode for TFTR L-mode discharge 49982 at $t=4.00 \mathrm{~s}$ with electrons and background ions only, from 2D results ${ }^{11,12}$, and from 1D (ballooning-representation) FULL code without $\left[\theta_{0}=0\right]$ and with $\left[\theta_{0}=\theta_{0}(M)\right]$ eigenfunction radial shearing effect, at $r / a=0.475$, for (a) $0 \leq M \leq 0.07$ and (b) $0 \leq M \leq \mathbf{0 . 8}$. 
$\left.(0.02)^{2}\right]^{1 / 4}$. The two-dimensional (2D) results (averaged over positive and negative Mach numbers) from Refs. 11 and 12 are plotted here in Fig. 12(a), along with the corresponding onedimensional (1D) ballooning-formalism results from the FULL code using the same model MHD equilibrium for $r / a=0.475$, for $\theta_{0}=0$ and $\theta_{0}=\theta_{0}(M)$. The 1D curve for $\theta_{0}=0$ corresponds to the toroidal rotation model without the additional effect of eigenfunction radial shearing, and shows very little effect on the growth rate for $M(r / a=0.475) \leq 0.07$. On the other hand, the 1D $\theta_{0}=\theta_{0}(M)$ curve, which models the additional effect of eigenfunction radial shearing, approximates the 2D curve well, and both show substantial stabilization of this mode at moderate Mach numbers. The extension of these 1D curves to larger Mach numbers is shown in Fig. 12(b). Without the eigenfunction shearing effect $\left(\theta_{0}=0\right)$, increasing local Mach number is initially somewhat destabilizing, but then becomes strongly stabilizing; the mode is completely stabilized only for $M \sim 0.8$. With the eigenfunction radial shearing effect $\left[\theta_{0}=\theta_{0}(M)\right]$, increasing local Mach number is immediately strongly stabilizing; the mode is completely stabilized for $M \sim 0.2$. Thus, in at least some cases this eigenfunction radial shearing effect can make a large difference in the critical amount of toroidal rotation for complete stabilization.

This approximate toroidal rotation model, without and with the additional eigenfunction radial shearing effect, can be applied to the same TFTR ERS case for discharge 84011 at $t=2.70 \mathrm{~s}$ (just before the ERS transition time) considered in Sec. III, for $r / a=0.20$. Here, the FULL code results are obtained for the electrostatic toroidal drift mode including a carbon impurity species and a slowing-down hot beam species, and using the numerically-calculated MHD equilibrium for the other parts of the instability calculation. Varying $M$ artificially, and maximizing $\gamma$ over $n$ (for $n=n_{\max }$ ) separately for each value of $M$, the results shown in Fig. 13 are obtained. For $\theta_{0}=0, \gamma$ and the associated $n_{\max }$ decrease slowly with increasing $M$. On the other hand, for $\theta_{0}=\theta_{0}(M)$, $\gamma$ decreases quickly for $M<0.1$, and then decreases slowly for $M>0.1$; the associated values of $n_{m a x}$ can also be substantially lower than for $\theta_{0}=0$. For the experimentally measured value of $V_{t o r}$ (measured spectroscopically for carbon), $\gamma$ is reduced by a factor of about three and $n_{\max }$ by a factor of about two by the eigenfunction radial shearing effect. Thus, in this case, substantial but 


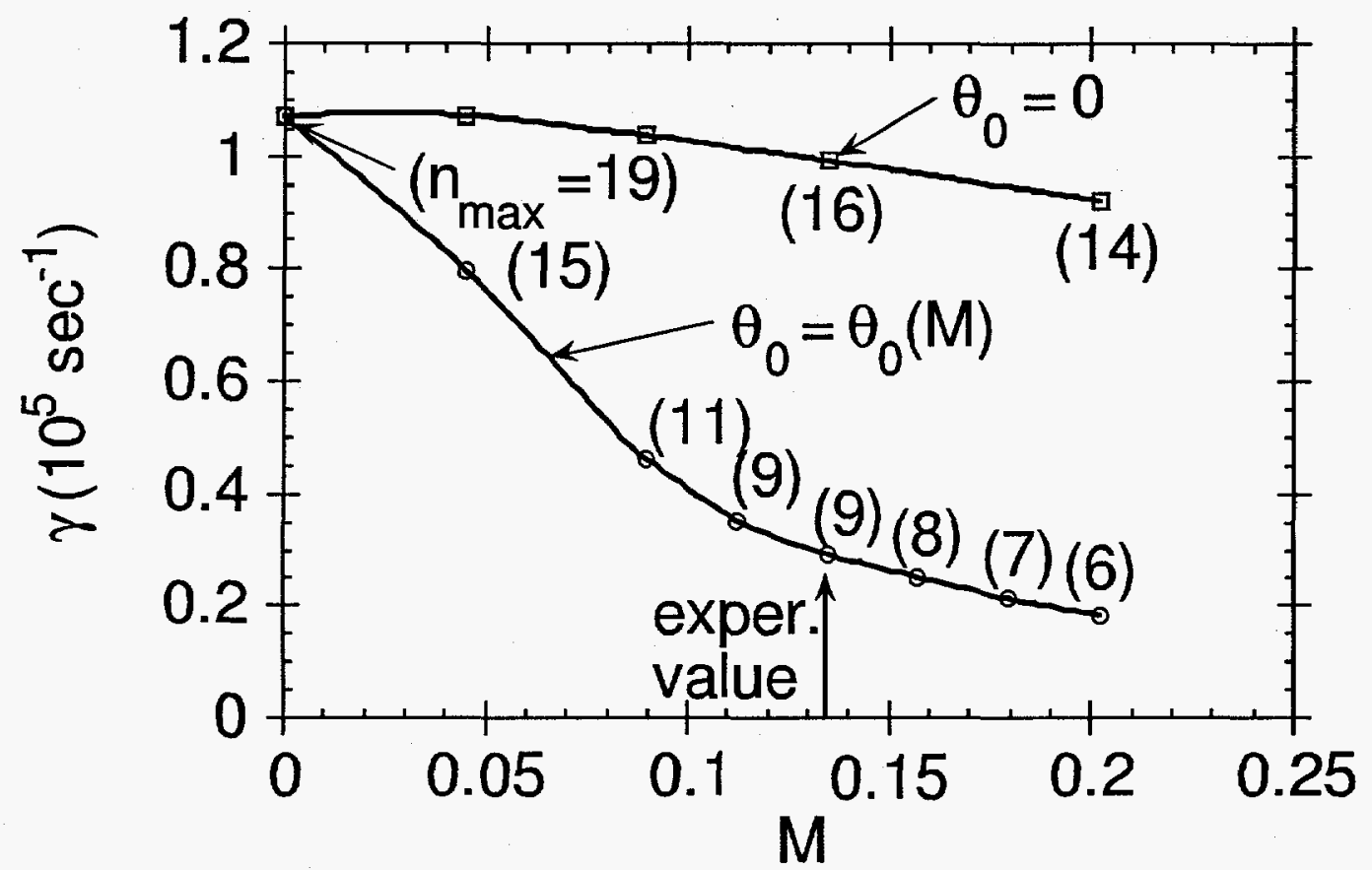

.FIG. 13. Results from FULL code for $\gamma$ versus $M$ for TFTR ERS discharge 84011 at $t=2.70$ $s$ and $r / a=0.20$, for electrostatic toroidal drift mode with carbon and slowing-down beam, with $\gamma$ maximized over $k_{\theta} \rho_{i}$ or $n$.

not complete stabilization is caused by toroidal rotation due to the eigenfunction radial shearing effect, according to the present model.

The overall stabilizing effects due to toroidal rotation can also be investigated for this TFTR ERS case as a function of time. We choose the radius $r / a=0.225$ where the toroidal rotation frequency $\Omega \equiv V_{\text {tor }} / R$ is a maximum at $t=2.70 \mathrm{~s}$, from the carbon spectroscopic experimental measurements; $\Omega$ and $\gamma_{E}$ are shown as functions of time at this radius in Fig. 14(a). The FULL code results, calculated as before with $\theta_{0}=\theta_{0}(M)$, for $\gamma$ versus $t$ with the associated values of $n_{\max }$, are shown in Fig. 14(b). There is an initial decrease in $\gamma$ from $t=2.5 \mathrm{~s}$, when the neutral beams turn on, to $t=2.6 \mathrm{~s}$, with a corresponding decrease in $n_{m a x}$, as $\Omega$ increases. But then from $t=2.6 \mathrm{~s}$ to $t=2.7 \mathrm{~s}, \gamma$ stays almost constant. Only after the ERS transition around $t=2.7 \mathrm{~s}$ does $\gamma$ decrease strongly again. This decrease is an effect, and not a cause, of the ERS confinement transition, as was discussed in Sec. III. Thus, sheared toroidal rotation of the bulk plasma does not seem to be sufficient by itself to account for the ERS transition, as might be the case if it 

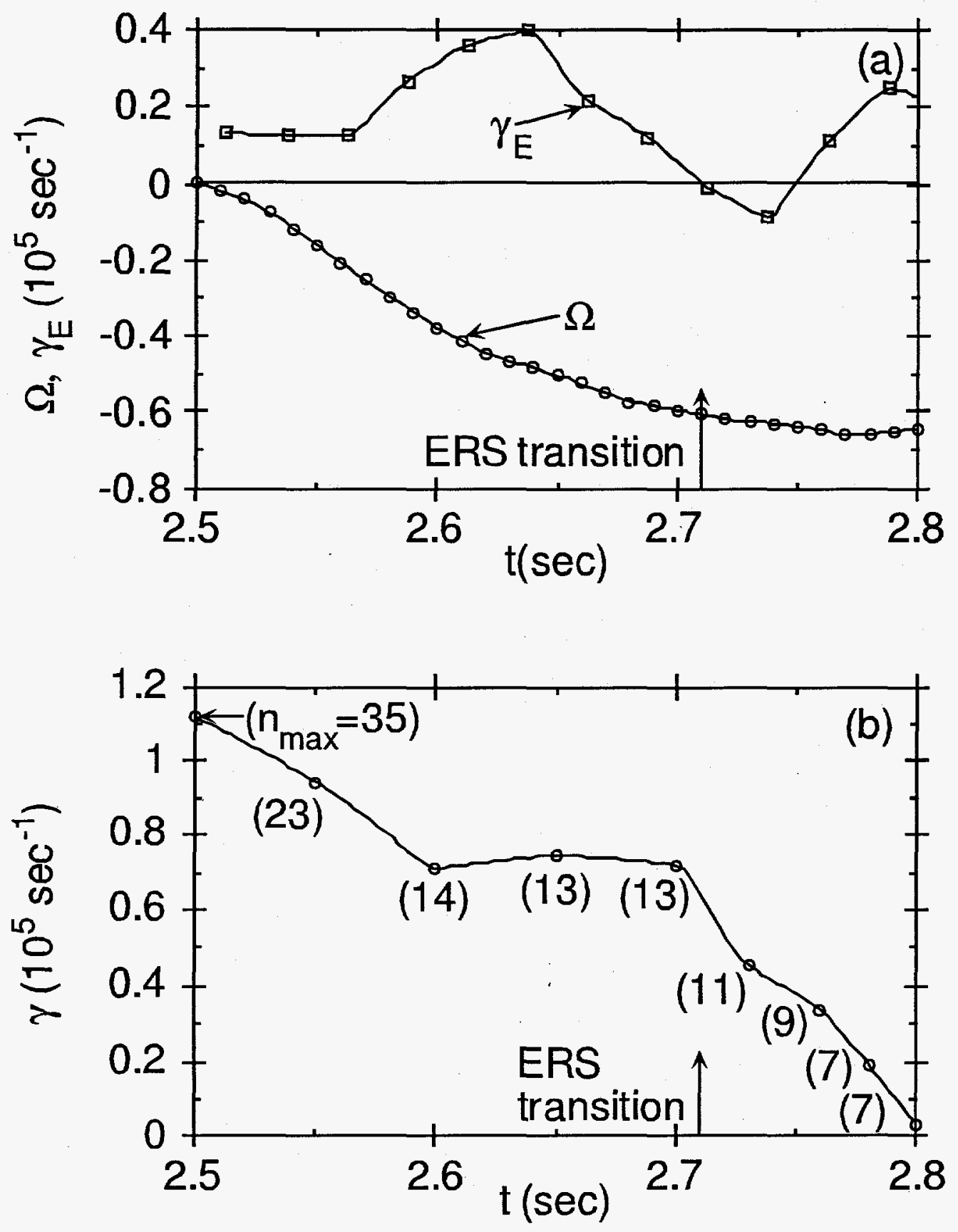

.FIG. 14. (a) $\gamma_{E}$ and $\Omega \equiv V_{\text {tor }} / R$ and (b) $\gamma$ versus $t$ for TFTR ERS discharge 84011 at $r / a=0.225$. Results for $\gamma$ from FULL code for toroidal drift mode with carbon and slowing down beam, maximized over $k_{\theta} \rho_{i}$ or $n$, including toroidal rotation model with eigenfunction radial shearing effect $\left[\theta_{0}=\theta_{0}(M)\right]$. 
completely stabilized the mode and thereby eliminated its associated anomalous transport. Also, $\gamma_{E}$ would presumably be having no direct effect locally at the ERS transition time, since it is going through zero then, as seen in Fig. 14(a). However, nonlocal nonlinear effects of the sorts discussed qualitatively in Ref. 23 could still be responsible for the observed transition.

\section{CONCLUSIONS}

A number of TFTR and DIII-D plasmas with negative or reversed central magnetic shear have been analysed using the FULL code for high- $n$ electrostatic toroidal drift modes. Realistic experimental profile data and numerically-calculated MHD equilibria were used to examine a variety of cases. Physically, changing the $q$-profile can affect the linear growth rates through at least four distinguishable mechanisms: (1) reduction of "bad curvature", (2) ion Landau damping, (3) ion sound term and ion temperature gradient mechanism strength, and (4) Shafranov shift. The overall effect on the instability is a combination of these things. For all of the TFTR and DIII-D negative central shear discharges examined here, and for several others, the direct changes with time in the width of the central stable region adjacent to the magnetic axis are governed more by changes in the density and temperature profiles than by changes in the $q$-profile, in the absence of rotation. The growth rate profiles change only gradually with time, on the transport time scale, as the density and temperature profiles change. In this sense, the changes seen here for the linear growth rates are an effect, not a cause, of the confinement transition. Thus, the "trigger" for the confinement transitions remains to be found. One possibility is the nonlinear decorrelation frequency $\gamma_{E}=\left|\left(R B_{\theta} / B\right) d / d r\left(E_{r} / R B_{\theta}\right)\right|$. For instance, comparing similar ERS and non-ERS discharges in TFTR, the linearly stable regions out from the magnetic axis are of almost the same width, but the larger values of $\gamma_{E}$ in the ERS discharge would be expected to give a wider nonlinearly stabilized region, consistent with the experimental measurements of a wider region of very low fluctuation level.

The linear effects of sheared toroidal rotation have been included here very approximately by means of a particular toroidal rotation model. The stabilizing effect due to radial shearing 
of the eigenfunction previously seen in a two-dimensional calculation can be modelled in a onedimensional, ballooning formalism calculation by means of a suitably chosen functional form for $\theta_{0}(M)$. With the form of $\theta_{0}(M)$ used here, complete stabilization of the low- $n$ trapped-ion mode for parameters of a TFTR L-mode discharge occurs for much lower values of $M$ than for $\theta_{0}=0$, that is, in the absence of the eigenfunction radial shearing. In TFTR ERS discharge 84011 , this model for the eigenfunction radial shearing can cause substantial reduction of the linear growth rates and corresponding lowering of $n_{\max }$. However, at one radius in the region where the experimental confinement transition is observed, progressive stabilization of the mode with time is seen, but no complete stabilization by the ERS transition time.

From these results, it is clear that linear theory by itself is insufficient to explain the relatively fast confinement transitions seen in TFTR and DIII-D, since linear growth rates, which depend on the equilibrium profiles and the MHD equilibrium, can only change on the transport time scale. Some additional nonlinear effect such as the $\gamma_{E}$ stabilization is needed to give a faster transition. This in turn could require that $E_{r}$ make a fast, perhaps driven, transition, for instance between two $E_{r}$ roots of a bifurcated state that results from a feedback loop. A conceptual framework for this kind of bifurcation model in a feedback situation is described in Ref. 23. It is hoped that the present sorts of linear and quasilinear calculations can be coupled into this more general framework.

In future work, it is clear that an improved rotational model would be desirable. In particular, a rotation model is needed which also includes the pressure gradient and poloidal rotation contributions to the radial electric field, not just the toroidal rotation contribution, and which includes general geometry for the magnetic surfaces. Also, if possible, a rotation model which unifies the treatment of bulk plasma rotation and of the nonlinear decorrelation frequency $\gamma_{E}$ would be desirable. Such a rotation model has been proposed in general terms in Ref. 24, and it will be investigated in future work. In addition, exploration of kinetic electromagnetic modes, such as the kinetically-calculated MHD ballooning mode, for instance in high- $\beta_{p}$ (large $p^{\prime}$ ) regimes, would be desirable. 


\section{ACKNOWLEDGMENTS}

This work was supported by United States Department of Energy Contract Nos. DE-AC03-

89ER51114 and DE-AC02-76-CHO-3073.

\section{DISCLAIMER}

This report was prepared as an account of work sponsored by an agency of the United States Government. Neither the United States Government nor any agency thereof, nor any of their empleyees, makes any warranty, express or implied, or assumes any legal liability or responsibility for the accuracy, completeness, or usefulness of any information, apparatus, product, or process disclosed, or represents that its use would not infringe privately owned rights. Reference herein to any specific commercial product, process, or service by trade name, trademark, manufacturer, or otherwise does not necessarily constitute or imply its endorsement, recommendation, or favoring by the United States Government or any agency thereof. The views and opinions of authors expressed herein do not necessarily state or reflect those of the United States Government or any agency thereof. 